\title{
Growth of N-polar GaN on vicinal sapphire substrate by metal organic chemical vapor deposition
}

\author{
Can-Tao Zhong, Guo-Yi Zhang*
}

Received: 17 October 2012/Revised: 15 March 2013/Accepted: 25 March 2013/Published online: 5 December 2013

(c) The Author(s) 2013. This article is published with open access at Springerlink.com

\begin{abstract}
The growth and properties of N-polar GaN layers by metal organic chemical vapor deposition (MOCVD) were reported. It is found that N-polar GaN grown on normal sapphire substrate shows hexagonal hillock surface morphology. With the misorientation angles increasing from $0.5^{\circ}$ to $2.0^{\circ}$ toward the a-plane of the sapphire substrate, the number of the hillock becomes less and less and finally the surface becomes flat one on the sapphire substrate with the misorientation angle of $2^{\circ}$. It is also found that the crystalline quality and the strain in the $\mathrm{GaN}$ are greatly influenced by the misorientation angle.
\end{abstract}

Keywords GaN; N-polarity; Metal organic chemical vapor deposition

\section{Introduction}

GaN-based III-nitrides are promising materials which have a lot of applications such as solid state lighting, full color display, laser printers, and high density information storage [1-3]. It is known that GaN has hexagonal crystal structure which shows polar nature. This polar nature has great influence on the growth behaviors and electrical and optical properties [4-6]. For example, most of $\mathrm{GaN}$ are epitaxially grown on c-sapphire substrate which leads to either metal(Ga)-polarity or N-polarity. Owing to the better stability of Ga-polarity and/or smooth surface, most of devices such as light-emitting diodes, laser diodes, and high electron

C.-T. Zhong, G.-Y. Zhang*

State Key Laboratory of Artificial Microstructure

and Mesoscopic Physics, School of Physics, Peking University,

Beijing 100871, China

e-mail: gyzhang@pku.edu.cn mobility transistors (HEMTs) are fabricated on Ga-polarity structure. Moreover, N-polarity GaN also shows its advantages. For example, in the case of HEMTs, N-polarity GaN shows low ohmic contact resistances and reduced short channel effect since the polarization direction is opposite to that of Ga-polarity one [7]. More importantly, the enhancement mode transistor can be realized by using the N-polar GaN-based heterostructure [7]. It was also reported that polarization induced hole doping in compositionally graded AlGaN grown on GaN along the N-polarity direction, which can enhance the p-type doping efficiency and thus leads to great application in light emitting devices, particularly at deep ultraviolet region [8]. In addition, both theoretical and experimental studies show that N-polarization emitters show several advantages over conventional emitters for device parameters such as electron overflow, injection efficiency, and laser threshold current density [9-11].

Unfortunately, to grow epitaxy of N-polarity GaN and its device structure were much less studied in comparison with that of Ga-polarity one. This is partially due to that it is quite difficult to grow the pure N-polarity GaN layer on sapphire substrate, the most popular substrate for nitride epitaxy. Thus, hillock growth morphology is usually observed on N-polar GaN which leads to difficultly achieve quantum structures with sharp interface. Therefore, most studies use either C-polarity $\mathrm{SiC}$ substrate or $\mathrm{N}$-polarity $\mathrm{GaN}$ bulk substrate, which are quite expensive and not suitable for mass production. In this paper, the growth of flat N-polarity GaN layers by using vicinal sapphire substrate was reported.

\section{Experimental}

GaN layers were grown by metal organic chemical vapor deposition (MOCVD, Axtron) system. Normal sapphire and 
those with misorientation angels of $0.5^{\circ}, 1.0^{\circ}$, and $2.0^{\circ}$ were used as substrates. Trimethylgallium (TMGa) and ammonia $\left(\mathrm{NH}_{3}\right)$ precursors were used for $\mathrm{Ga}$ and $\mathrm{N}$ sourses, respectively. $\mathrm{H}_{2}$ was used as carrier gas and the reactor pressure was $1.01 \times 10^{-6} \mathrm{~Pa}$. The key point to achieve N-polarity GaN samples was using nitridation process, where the sapphire substrate was exposed to the $\mathrm{N}$ precursor at $1,100^{\circ} \mathrm{C}$ for 2-3 min after thermal annealing. An AlN ultrathin layer with $\mathrm{N}$-polarity is believed to be formed by this nitridation process, which is similar to the case of relative long time sapphire nitridation process in the molecular beam epitaxy (MBE) [12]. Then low temperature GaN buffer layer with a thickness of $30 \mathrm{~nm}$ and high temperature $\mathrm{GaN}$ epilayer with a thickness of about $2 \mu \mathrm{m}$ were grown in sequence. The surface morphology of the samples was measured with an optical microscope (OM). Atomic force microscopy (AFM) was also used to give us the surface roughness (root-meansquare value). Crystalline quality of the samples was investigated by X-ray diffraction (XRD), where the $\omega$ rocking curve of both the symmetric and asymmetric scans are measured. Raman measurement was also performed to investigate the stain status in the samples.

\section{Results and discussion}

Figure 1 shows the OM images of GaN samples grown on sapphire substrate with different mis-orientation angles. It is shown that hexagonal hillock surface morphology is observed in GaN films grown on normal sapphire substrate. The size of each hillock is about $150 \mu \mathrm{m}$, which is the usual surface for the N-polar GaN layers grown by MOCVD, as reported by several groups $[13,14]$. It is believed that the formation of this kind of big hexagonal hillock structure is due to that the N-polarity is not perfect, i.e., some parts or micrograins show Ga-polarity $[13,14]$. Since the growth rate of Ga-polarity is a little higher than that of $\mathrm{N}$-polarity, the big hexagonal grains are formed centered by the Gapolarity grain in nanoscale. It is shown that the number of hexagonal hillock grains becomes less with the mis-orientation angle increasing to $0.5^{\circ}$, where some areas show half or part of hexagonal grains. When the mis-orientation angle increases to $1.0^{\circ}$, the density of grains becomes much smaller while the grain size also becomes smaller. The surface finally changes to flat one and is free of hexagonal grain when the mis-orientation angle increases to $1.0^{\circ}$. Figure 2 shows the surface morphology of $\mathrm{GaN}$ layers investigated by AFM, which shows surface features in much small area in comparison with that shown in Fig. 1. Figure 2a shows the morphology of area free of hillock for GaN layer grown on $0.5^{\circ}$-off sapphire. The surface in these areas is quite flat with rms roughness of as small as $0.6 \mathrm{~nm}$ in $10 \mu \mathrm{m} \times 10 \mu \mathrm{m}$ scanned area. This value decreases to $0.35 \mathrm{~nm}$ in $3 \mu \mathrm{m} \times 3 \mu \mathrm{m}$ scanned area. It is interesting that there is no step, which is different from the step-flow growth of Ga-polar GaN [15]. This shows advantages to
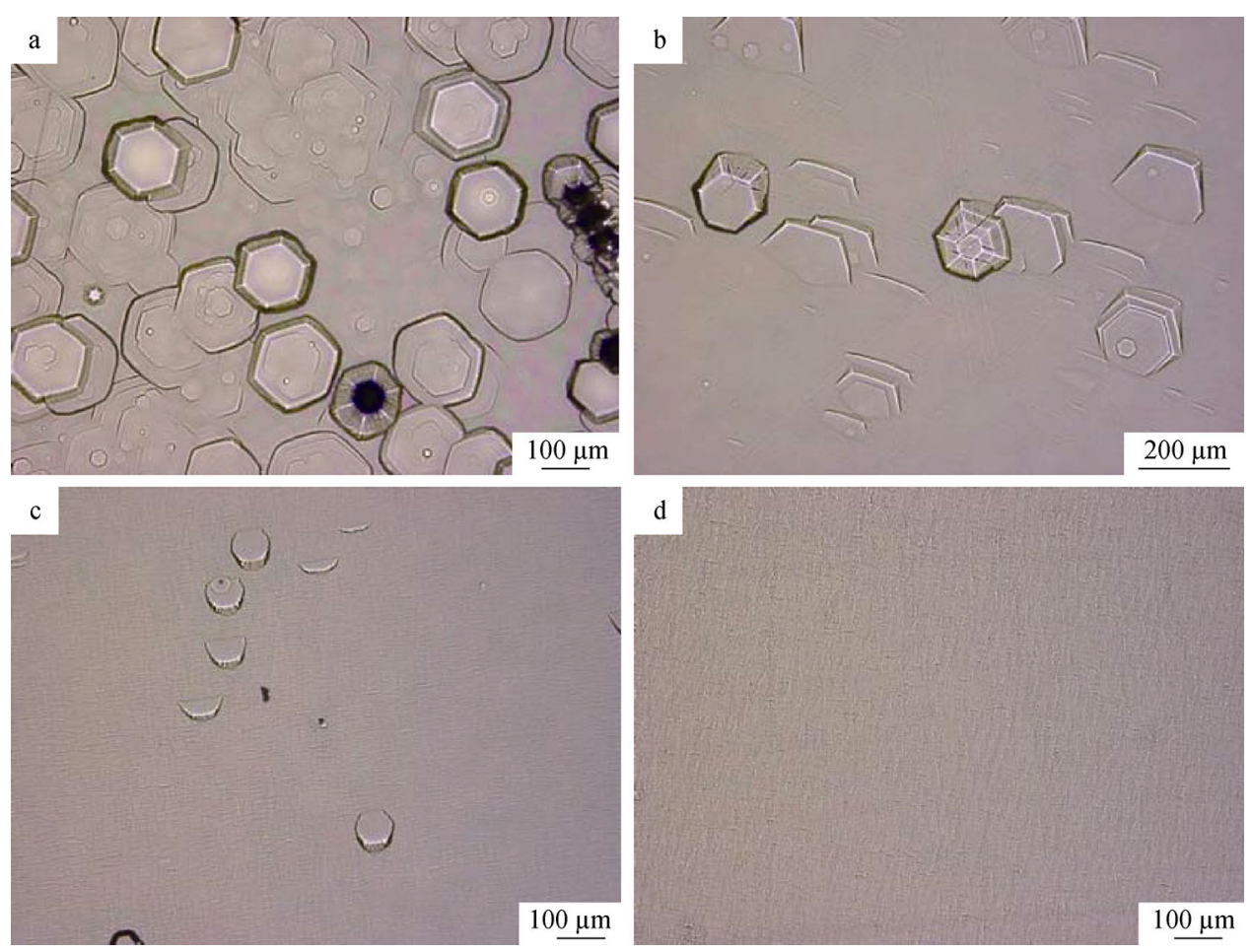

Fig. $1 \mathrm{OM}$ images of GaN samples grown on sapphire substrate with different mis-orientation angles: a on normal sapphire, b on $0.5^{\circ}$-off sapphire, $\mathbf{c}$ on $1.0^{\circ}$-off sapphire, and $\mathbf{d}$ on $2.0^{\circ}$-off sapphire 
realize quantum wells with very sharp interface and benefits the emission efficiency. With the mis-orientation angle increasing, step-bunching is observed in the area free of hexagonal grains. And the surface becomes rougher and rougher due to that the misorientation angle becomes larger. Within each terrace, the surface is still flat but the rms roughness becomes larger with mis-orientation angle increasing. In addition, these steps are along the direction of $\langle 1-100\rangle$ of the GaN.

Figure 3 shows the full widths at half maximum (FWHMs) of XRD $\omega$-rocking curves for symmetric (002) and asymmetric (102) planes for the $2 \mu \mathrm{m}$ thick N-polar $\mathrm{GaN}$ layers grown with different misorientation angles. The FWHM of (002) $\omega$-scans is about 100 arcsec, which is almost not influenced by the mis-orientation except that it is a little bit larger for GaN film with mis-orientation of $2.0^{\circ}$. In addition, the FWHM of (102) $\omega$-scans is about 700 arcsec for $\mathrm{GaN}$ layer grown on normal sapphire substrate. It increases to about 1,000 arcsec with the mis-orientation angle increasing to $0.5^{\circ}$, and then gradually decreases with the mis-orientation angle further increasing. It is known that the broadening of (002) $\omega$-scan is mainly due to the threading dislocations (TDs) with screw component (tilt) and the broadening of (102) $\omega$-scan is mainly ascribed to the TDs with edge component (twist). Therefore, it shows in Fig. 3 that $\mathrm{GaN}$ film grown at $0.5^{\circ}$-off sapphire shows the highest density of TDs with edge component and the increase of mis-orientation angle of sapphire substrate leads to the reduction of edge-type TDs density.

Figure 4 shows Raman spectra of GaN layers grown on sapphire substrate with different mis-orientation angles, which is measured in back-scattering geometry. As shown in Fig. 4, two peaks are observed for each sample. The dominant peaks are located at around $565 \mathrm{~cm}^{-1}$ which is ascribed to $E_{2}$ (high) mode of GaN. Other peaks located at around $751 \mathrm{~cm}^{-1}$ are assigned to $E_{\mathrm{g}}$ mode of sapphire substrate. It is known that the residual strain of epitaxial layers can be estimated from the scattering peak of $E_{2}$ (high) mode, while the FWHMs of $E_{2}$ (high) mode scattering peaks can be used as a merit of crystalline quality. Figure 5 shows the Raman peak of $E_{2}$ (high) mode and its FWHM for the GaN layers grown on sapphire with different mis-orientation angles. It is shown that the Raman frequency of the $E_{2}$ (high) mode changes from 565.4 to $564.5,566.0$, and $566.7 \mathrm{~cm}^{-1}$ for GaN layers grown on normal sapphire substrate and $0.5^{\circ}, 1.0^{\circ}$, and $2.0^{\circ}$ off sapphire substrate. In comparison with the Raman frequency of the $E_{2}$ (high) mode for strain-free $\mathrm{GaN}$, it is shown that almost all the $\mathrm{GaN}$ is undertaken compressive strain, while the sample grown on $0.5^{\circ}$-off sapphire substrate is almost strain free [16]. Then, with the mis-orientation angle further increasing, the compressive strain
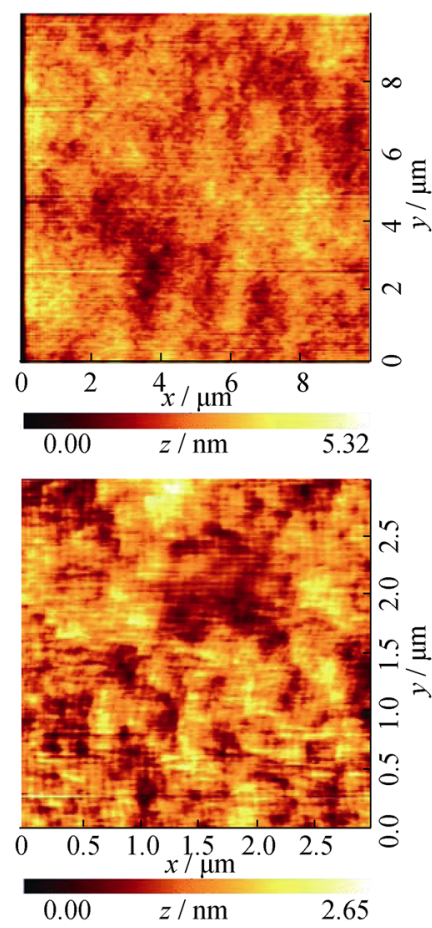

a
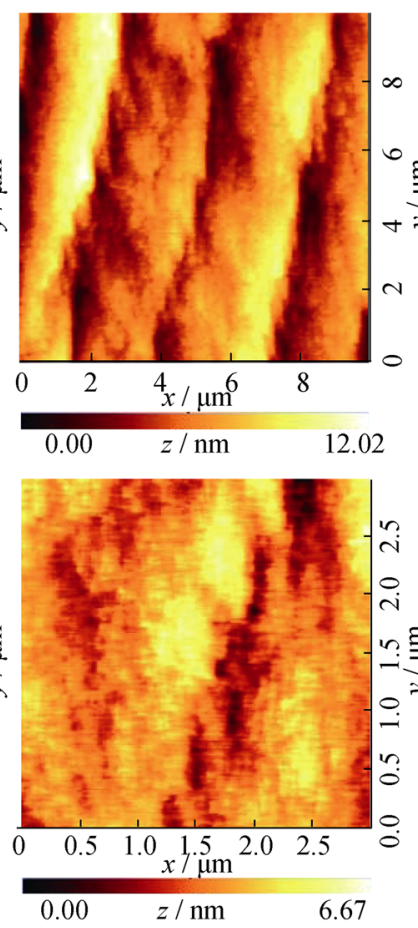

b
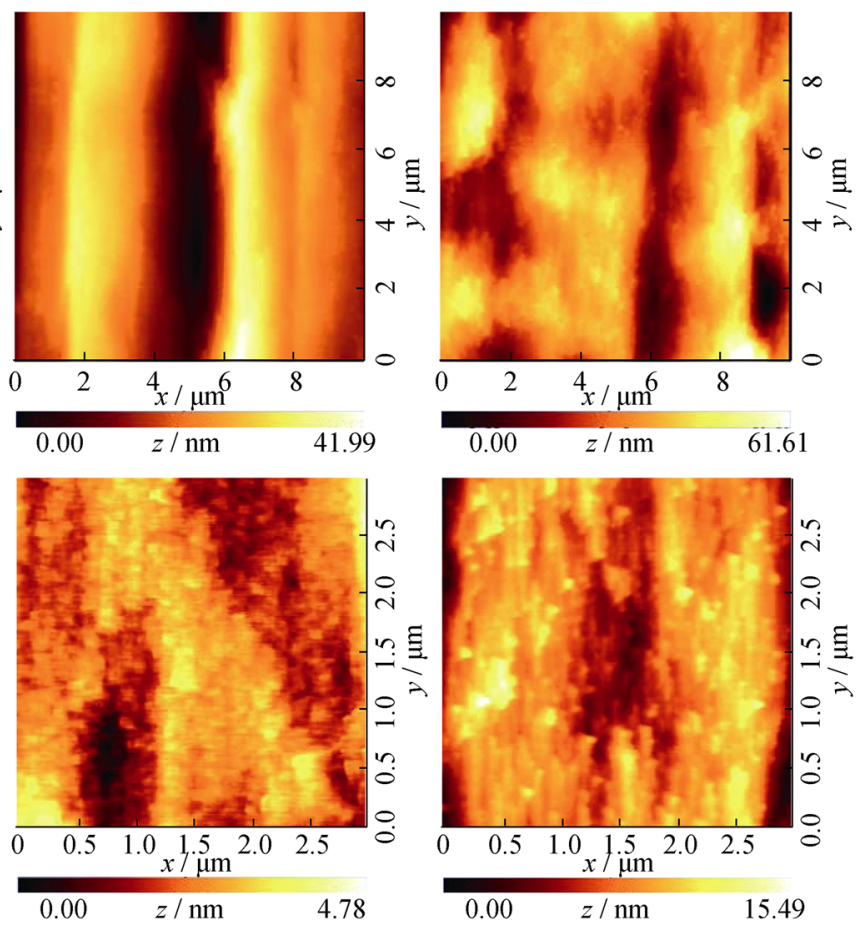

$\mathrm{c}$

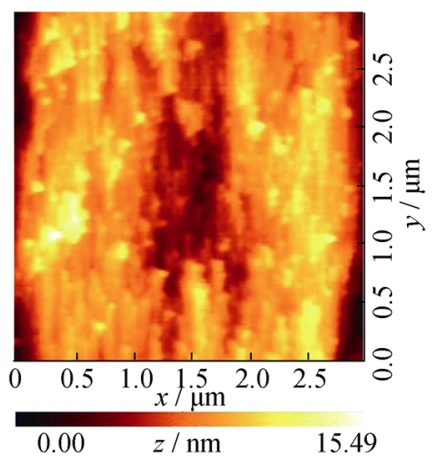

d

Fig. 2 AFM images of GaN samples grown on sapphire substrate with different mis-orientation angles: a on normal sapphire, b on $0.5^{\circ}$-off sapphire, $\mathbf{c}$ on $1.0^{\circ}$-off sapphire, and $\mathbf{d}$ on $2.0^{\circ}$-off sapphire. Scanned areas being $10 \mu \mathrm{m} \times 10 \mu \mathrm{m}$ and $3 \mu \mathrm{m} \times 3 \mu \mathrm{m}$ 


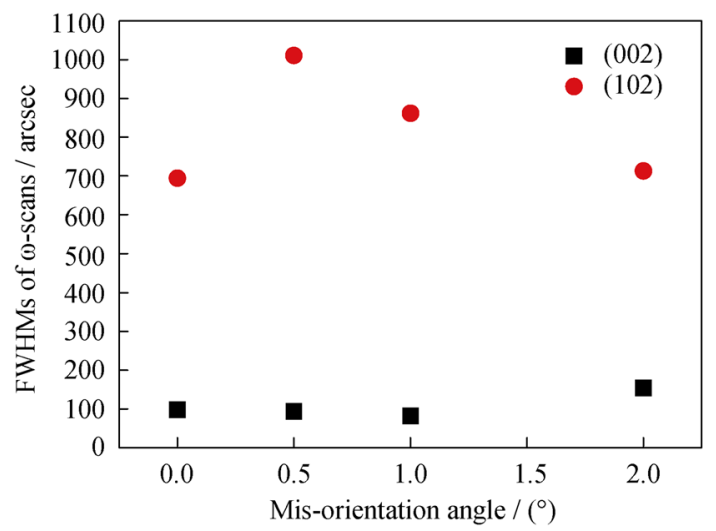

Fig. 3 FWHMS of $\omega$-rocking curves for (002) symmetric and (102) asymmetric planes of $\mathrm{GaN}$ samples grown on sapphire substrate with different mis-orientation angles

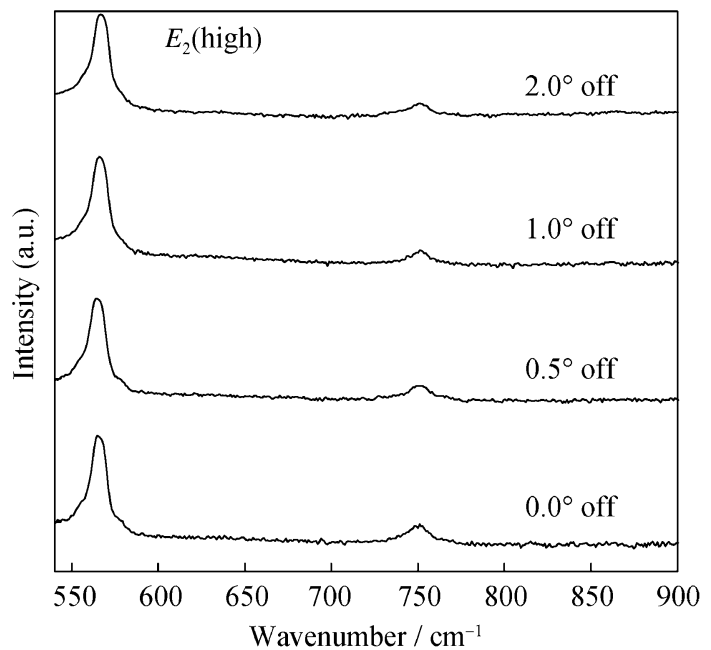

Fig. 4 Raman scattering spectra of GaN samples grown on sapphire substrate with different mis-orientation angles

becomes larger and larger. In Fig. 5, it is also shown that the FWHMs of the Raman scattering peak of the $E_{2}$ (high) mode increases from 6.16 to $6.41 \mathrm{~cm}^{-1}$, and then decreases to $5.98 \mathrm{~cm}^{-1}$ with the mis-orientation angles increasing. This indicates that the crystalline quality of $\mathrm{GaN}$ layer becomes worse first and then is gradually enhanced with the mis-orientation angle increasing, which coincides with the tendency of the edge-type TDs as a function of the misorientation angle well as shown in Fig. 3. In Fig. 5, it can be seen that the tendency of residual strain and crystalline quality is very similar, i.e., the poorer the crystalline quality is, the smaller the residual strain is. This indicates that the threading dislocation can effectively release the strain in GaN where the GaN with the largest TDs density has the smallest residual compressive strain.

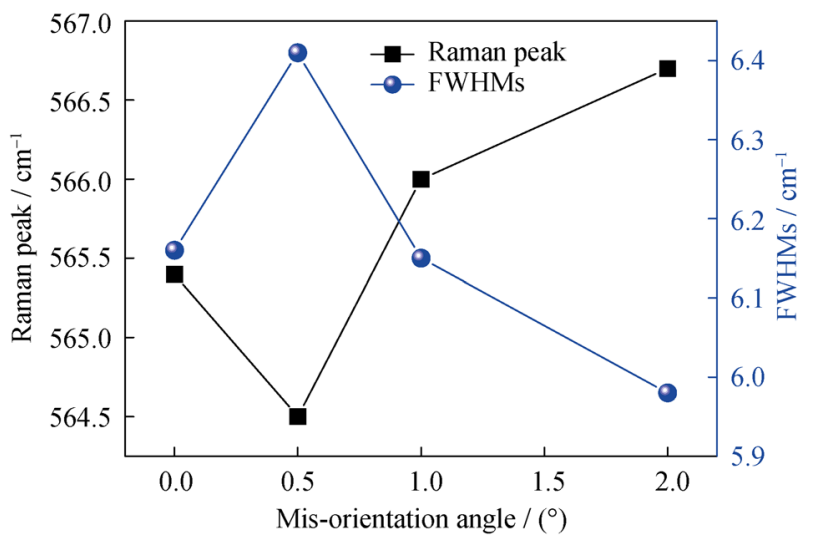

Fig. 5 Raman scattering peaks of $E_{2}$ (high) mode and their FWHMs for $\mathrm{GaN}$ layers grown on sapphire substrate with different misorientation angles

\section{Conclusion}

In summary, N-polarity GaN layers were grown on sapphire substrate by MOVCD. And the flat surface was successful obtained by using the mis-orientation angle sapphire substrate. The hillocks become less and less with the mis-orientation angles increasing and are finally eliminated and resulted in flat surface at the mis-orientation angle of $2.0^{\circ}$. The crystalline quality is firstly reduced for $\mathrm{GaN}$ films grown on the mis-orientation angle of $0.5^{\circ}$ and then gradually improved with the mis-orientation angle further increasing, as revealed by the XRD and Raman measurement. It is shown in this work that the application of mis-orientation angle substrate is a successful way to obtain the flat $\mathrm{GaN}$ with $\mathrm{N}$-polarity and to improve the crystalline quality as well.

Acknowledgments This work was financially supported by the National Basic Research Program of China (Nos. 2012CB619303 and 2012CB619304), the National Natural Science Foundation of China (Nos. 11023003, 10990102, 11174008 and 61076012), and the National High Technology Research \& Development Project of China (No. 2011AA03A103).

Open Access This article is distributed under the terms of the Creative Commons Attribution License which permits any use, distribution, and reproduction in any medium, provided the original author(s) and the source are credited.

\section{References}

[1] Takahashi K, Yoshikawa A, Sandhu A. Wide Bandgap SemiConductors. Berlin: Springer; 2007. 2.

[2] Morkoc H. Nitrides Semiconductors and Devices. Berlin: Springer; 1999. 5.

[3] Wang X, Yoshikawa A. Molecular beam epitaxy growth of GaN, AIN and InN. Prog Cryst Growth Charact Mater. 2004;48-49:42. 
[4] Hellman ES. The polarity of GaN: a critical review. MRS Internet J Nitride Semicond Res. 1998;3(11):11.

[5] Sumiya M, Fuke S. Review of polarity determination and control of GaN. MRS Internet J Nitride Semicond Res. 2004;9(1):1.

[6] Stutzmann M, Ambacher O, Eickhoff M, Karrer U, Lima Pimenta A, Neuberger R, Schalwig J, Dimitrov R, Schuck PJ, Grober RD. Playing with polarity. Phys Status Solidi B. 2001;228(2):505.

[7] Rajan S, Chini A, Wong MH, Speck JS, Mishra UK. N-polar GaN/AlGaN/GaN high electron mobility transistors. J Appl Phys. 2007;102(4):044501.

[8] Simon J, Protasenko V, Lian C, Xing H, Jena D. Polarizationinduced hole doping in wide-band-gap uniaxial semiconductor heterostructures. Science. 2010;327(5961):60.

[9] Kuo YK, Horng SH, Yen SH, Tsai MC, Huang MF. Effect of polarization state on optical properties of blue-violet InGaN light-emitting diodes. Appl Phys A. 2010;98(3):509.

[10] Yen SH, Kuo YK. Polarization-dependent optical characteristics of violet InGaN laser diodes. J Appl Phys. 2008;103(10): 103115.

[11] Akyol F, Nath DN, Krishnamoorthy S, Park PS, Rajan S. Suppression of electron overflow and efficiency droop in N-polar GaN green light emitting diodes. Appl Phys Lett. 2012;100(11):111118.
[12] Wang X, Iwaki H, Murakami M, Du X, Ishitani Y, Yoshikawa A. Molecular beam epitaxy growth of single-domain and highquality $\mathrm{ZnO}$ layers on nitrided (0001) sapphire surface. Jpn J Appl Phys. 2003;42(2A):L99.

[13] Weyher JL, Brown PD, Zauner ARA, Muller S, Boothroyd CB, Foord DT, Hageman PR, Humphreys CJ, Larsen PK, Grzegory I, Porowski S. Morphological and structural characteristics of homoepitaxial GaN grown by metalorganic chemical vapour deposition (MOCVD). J Cryst Growth. 1999;204(4):419.

[14] Brown PD, Weyher JL, Boothroyd CB, Toord DT, Zauner ARA, Hageman PR, Larsen PK, Bockowski M, Humphreys CJ. Humphreys, inversion domain nucleation in homoepitaxial GaN. Inst Phys Conf Ser. 1999;164:381.

[15] Loren K, Gonsalves M, Kim W, Narayanan V, Mahajan S. Comparative study of GaN and AlN nucleation layers and their role in growth of $\mathrm{GaN}$ on sapphire by metalorganic chemical vapor deposition. Appl Phys Lett. 2000;77(21):3391.

[16] Dong Y, Song JH, Kim HJ, Kim TS, Ahn BJ, Song JH, Cho IS, Im WT, Moon Y, Hwang S, Hong SK, Lee SW. Raman and emission characteristics of $\alpha$-plane InGaN/GaN blue-green light emitting diodes on $\gamma$-sapphire substrates. Appl Phys. 2011;109(4):043103. 